УДК 621.37:519.21

\title{
ИНВАРИАНТ ГРУППЫ ОТОБРАЖЕНИЙ СЛУЧАЙНЫХ ВЫБОРОК В ВЫБОРОЧНОМ ПРОСТРАНСТВЕ СО СВОЙСТВАМИ РЕШЕТКИ
}

\author{
ПОПОВ А. А.
}

\author{
Центральный научно-исследовательский институт вооружения \\ и военной техники Вооруженных Сил Украины, \\ Украина, Киев
}

\begin{abstract}
Аннотация. Получена характеристика статистической взаимосвязи случайных выборок, инвариантная по отношению к группе их отображений, которая построена на выборочном пространстве со свойствами решетки. Исследуется возможность ее применения для анализа качества обработки изображений
\end{abstract}

Ключевые слова: выборочное пространство; выборка; инвариант; решетка; L-группа

Одной из существенных проблем оценки алгоритмов и устройств обработки изображений является выбор адекватного критерия оценки их эффективности. При решении некоторых задач обработки изображений, часть информации, которая в них содержится, в ряде случаев теряется. Это имеет место в задачах дискретизации и квантовании непрерывного сигнала изображения, преобразования изображений с использованием методов пространственной и/или частотной коррекции изображений, уменьшения разрядности цветовой палитры (динамический диапазон изображения), а также при сжатии изображений. Для оценки качества обработки изображений трансформированное из исходного изображение сравнивается с первоначальным при помощи какой-либо метрики.

В качестве меры близости выборочных данных $x, y$, составляющих пару изображений $X, Y$, используются известные метрики $d(x, y)$, которые характеризуют соответствующую меру отклонения значений элементов изображения (пикселов) $x_{i j} \in x, y_{i j} \in y[1-3]$ :

$$
\begin{gathered}
d_{0}(x, y)=\max _{i, j}\left|x_{i j}-y_{i j}\right| \\
d_{p}(x, y)=\left(\frac{1}{n m} \sum_{i} \sum_{j}\left|x_{i j}-y_{i j}\right|^{p}\right)^{1 / p}, \\
p \in N,
\end{gathered}
$$

где $N-$ множество натуральных чисел, $x=\|x(i, j)\|=\left\|x_{i j}\right\|, \quad y=\|y(i, j)\|=\left\|y_{i j}\right\|$ - выборки-матрицы двух изображений $X, \quad Y$; $i=1, \ldots, n, j=1, \ldots, m ;(i, j)$ - координаты элемента изображения.

Часто в практических приложениях используется мера близости, построенная на метрике (1б) при $p=2$, называемая пиковым отношением сигнал/шум PSNR (peak-signal-noise ratio) [3]:

$$
d_{\mathrm{PSNR}}(x, y)=10 \lg \left(\frac{A^{2} n m}{\sum_{i} \sum_{j}\left|x_{i j}-y_{i j}\right|^{2}}\right),
$$

где $A-$ уровень белого. 